\title{
Modeling Techniques of Nested Helical Structure Based Geometry for Numerical Analysis
}

\author{
Cengiz Erdönmez ${ }^{1, *}$ - Cevat Erdem İmrak ${ }^{2}$ \\ ${ }^{1}$ Istanbul Technical University, Institute of Informatics, Turkey \\ 2 Istanbul Technical University, Faculty of Mechanical Engineering, Turkey
}

\begin{abstract}
The aim of this paper is to introduce a new methodology of defining and modeling the nested helical structure (NHS) for wire ropes, and to present an accurate wire rope 3D solid modeling, which can be used for finite element analysis. Both single and nested helical wire parametric equations are presented. Derivation of the Frenet-Serret frame for the helical structures is explained, which enables one to define a normal plane along the centerline of a single helical or nested helical curve in $3 D$ space. Both single helical and nested helical solid structures are generated by sweeping a circle or a quadrilateral by using the moving trihedron along the centerline of the helical geometry. When the length of the NHS is increased, surface quality of the NHS diminishes rapidly and it is not possible to generate a good mesh by using the commercial CAD tools. However, the proposed method introduces a solution of modeling helical structures without length limitation with generating an accurate and valid mesh. Illustrative examples are presented to show the benefits of the proposed modeling procedure by using finite element analysis.

(C)2011 Journal of Mechanical Engineering. All rights reserved.
\end{abstract}

Keywords: nested helical structure, nested helix, single helix, double helix, Frenet-Serret frame

\section{INTRODUCTION}

Helix geometry is one of the interesting curves among the space curves in $3 \mathrm{D}$ spaces. Some structures such as screws, DNA molecules, and wire ropes have helical substructures. The general form of a helix can be referred to as a single helix. It is effortless to construct and model a single helical geometry using the commercial CAD software. Coiling a helix around another creates a new geometry, which can be called double helical or nested helical structure. The word double helix is not defined explicitly at the moment. Double helix is used mostly for the DNA molecules and structures in the literature [1] to [3]. A double helix typically consists of two similar helices with the same axis, differing by starting angle along the axis. Intertwined helices with different radii, i.e. successive layers vary in their radii to guarantee the maximum possible geometric distance in DNA molecules. For this reason, the complicated new type of helix which is nested over a single helix is called a nested helix (NH) throughout this paper so as to distinguish these two helical structures. The structures produced using $\mathrm{NH}$ are called nested helical structures (NHS). The use of helical wires can be seen widely in the construction of wire ropes. Mechanical and structural compositions are analyzed analytically in a number of papers [4] to [6].

Meanwhile, it is difficult to construct a NHS by using commercial CAD tools. In this paper, first a general definition of the helix is discussed and then the parametric representation of the $\mathrm{NH}$ is presented. A normal plane perpendicular to a curve is defined using the Frenet-Serret frame [7], which is known as the moving triad. Modeling a solid wire using NHS, which enables the production a valid meshed solid model to use during finite element analysis (FEA) is introduced. The aim of this paper is to exhibit a new methodology to model NHS and remove the difficulties encountered during modeling and numerical analysis of wire ropes. Finally, a wire strand and an independent wire rope core (IWRC) are modeled and analyzed using the proposed method.

\section{GENERAL DEFINITION OF HELICAL GEOMETRIES}

Helix as a space curve in $R^{3}$ is defined solely with the following Eq.:

*Corr. Author's Address: Institute of Informatics, Computational Science and Engineering Program, 


$$
\begin{aligned}
& x=r \cos \theta, \\
& y=r \sin \theta, \\
& z=p \frac{\theta}{2 \pi},
\end{aligned}
$$

where $\theta, r$, and $p$ represents the turning angle and radius and pitch length of the helix, respectively. A general helix geometry is depicted in Fig. 1. The helix angle $\alpha$ is related with the pitch length of the helix $p$ directly with $\tan \alpha=p /(2 \pi r)$. A helix with a length of $L$ can be generated by computing the number of as $\omega=L / p$ along its length where $p$ corresponds to the pitch length of the helix. The helix angle $\theta$ has to sweep an angle starting from $\theta=0$ to $\theta=\omega \cdot 2 \pi$ for producing a helical structure of length $L$. In this way, the desired helical curve is generated.

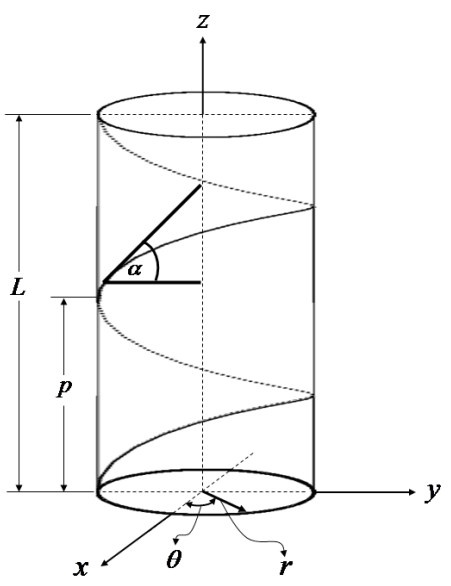

Fig. 1. General helix geometry

\subsection{Nested Helical Geometry}

Cartesian coordinate system $(x, y, z)$ with the Cartesian frame $\left\{e_{x}, e_{y}, e_{z}\right\}$ is used to define the location of the centerline of a single outer helix by:

$$
\begin{aligned}
& x_{s}=r_{s} \cos \theta_{s}, \\
& y_{s}=r_{s} \sin \theta_{s}, \\
& z_{s}=r_{s} \theta_{s} \tan \alpha_{s} .
\end{aligned}
$$

The helix lies along the $e_{z}$ axis while the radius of the single helix and laying angle are defined as $r_{s}$ and $\alpha_{s}$ respectively. Free angle $\theta$ defines the location of the wire around the rope axis $e_{z}$ relative to $e_{x}$ and the single helix phase angle is defined as $\theta_{0}=\theta_{(z=0)}$. Using the free angle $\theta$ and the phase angle $\theta_{0}$, helix angle of the single helical wire is defined as $\theta_{\mathrm{s}}=\theta_{0}+\theta$. Angle $\theta_{s}$ is used to define both the single helical wire angle of strand 1 and the outer single helical wire angle of the strand 2 as depicted in Fig. 2a.

The outer nested helical wires on strand 2 are wound around the outer single helical wire by using the centerline of the single helical wire defined in Eq. (2) by using the angle of $\theta_{d}$. Centerline of the nested helical geometry is depicted in Fig. $2 \mathrm{a}$ and defined by the following parametric Eq. [8]:

$$
\begin{gathered}
x_{d}=x_{s}+r_{d}\left(\cos \theta_{d} \cos \theta_{s}-\sin \theta_{d} \sin \theta_{s} \sin \alpha_{s}\right), \\
y_{d}=y_{s}+r_{d}\left(\cos \theta_{d} \sin \theta_{s}+\sin \theta_{d} \cos \theta_{s} \sin \alpha_{s}\right), \\
z_{d}=z_{s}-r_{d} \sin \theta_{d} \cos \alpha_{s},
\end{gathered}
$$

where $\theta_{d}=m \cdot \theta_{s}+\theta d_{0}$ is the $\mathrm{NH}$ wrapping angle around the single helical wire centerline while $\mathrm{m}$ is the construction parameter, which is the ratio of the nested helical wire rotating angle to the single helical wire rotation angle, $m=\theta_{d} / \theta_{s}$, and $\theta_{d 0}$ is the wire phase angle. The distance $r_{d}$ is defined by the distance between the NH centerline and the single helix centerline as shown in Fig. 2a. In addition, the solid model of the nested helical wires around a single helical wire centerline is presented in Fig. 2b. According to Eq. (3) a right lay $\mathrm{NH}$ can be constructed. To construct a left lay $\mathrm{NH}$, it is enough to negate one of the coordinate values of $x_{d}$ or $y_{d}$ which are defined in Eq. (3).

\subsection{A Moving Trihedron and Plane Construction}

Frenet-Serret expression describes the kinematic properties of a particle, which moves along a continuous, differentiable curve in 3D Euclidean space $R^{3}$. Frenet-Serret frame [7] is used to construct a normal plane, which is perpendicular to the single helical or NHS to construct a 3D solid part.

Let $I \subset R$ be an interval and $\psi: I \rightarrow R^{3}$ be a parameterized space curve, assumed regular and free of inflection points. $\psi(\theta)$ is the trajectory of a particle moving through $3 \mathrm{D}$ space. The moving trihedron, known as the Frenet-Serret frame, corresponds to an orthonormal basis of three vectors; $T(\theta), N(\theta)$ and $B(\theta)$. The unit tangent vector $T(\theta)$, the unit binormal vector $B(\theta)$ and the unit normal vector $N(\theta)$ can be defined respectively, 

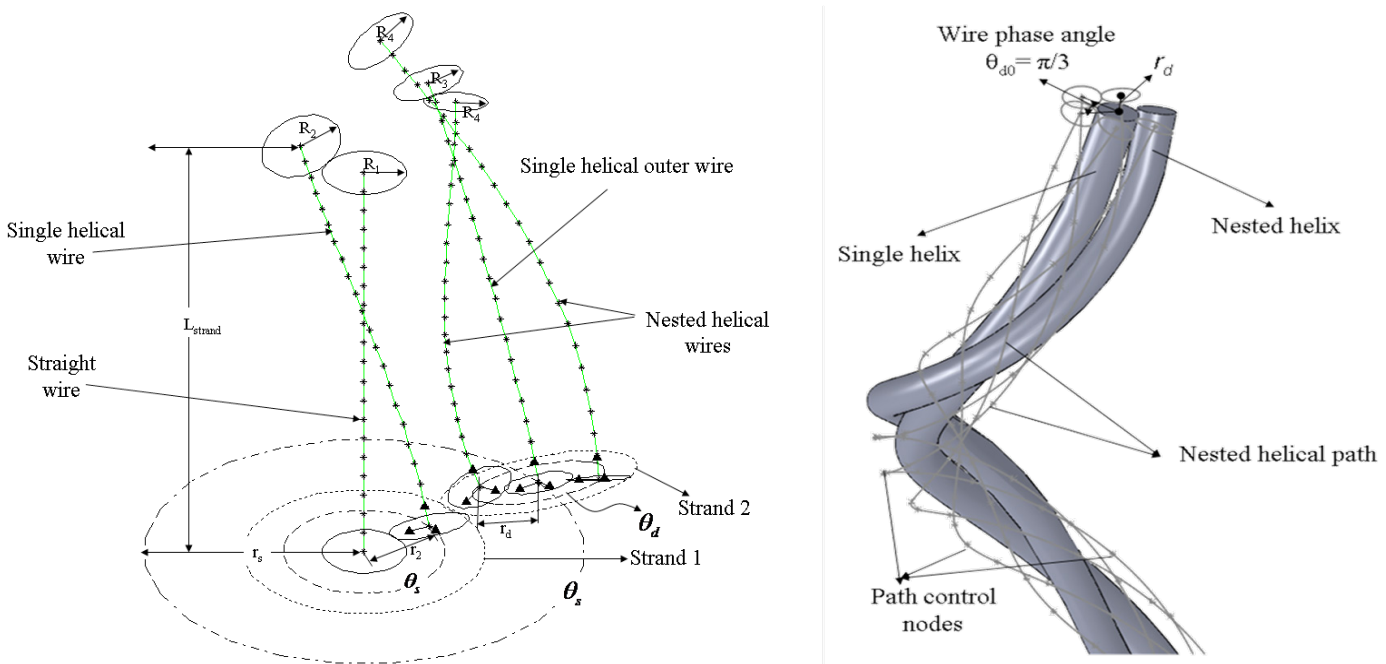

Fig. 2. IWRC; a) single and nested helical wire centerlines, b) solid model of the nested helical wire

$$
\begin{gathered}
T(\theta)=\frac{\psi^{\prime}(\theta)}{\left\|\psi^{\prime}(\theta)\right\|}, \\
B(\theta)=\frac{\psi^{\prime}(\theta) \times \psi^{\prime \prime}(\theta)}{\left\|\psi^{\prime}(\theta) \times \psi^{\prime \prime}(\theta)\right\|}, \\
N(\theta)=\frac{B(\theta) \times \psi^{\prime}(\theta)}{\left\|B(\theta) \times \psi^{\prime}(\theta)\right\|} .
\end{gathered}
$$

The line $\psi(\theta)+t T(\theta)$ is the tangent line at $\psi(\theta)$. Here $t \in R$ represents a parameter corresponding to create the tangent line in a given range. The binormal vector $B(\theta)$ is perpendicular to both $\psi^{\prime}(\theta)$ and $\psi^{\prime \prime}(\theta)$ and hence perpendicular to the osculating plane. The line $\psi(\theta)+t B(\theta)$ is the binormal line at $\psi(\theta)$. Finally, the normal vector is the vector perpendicular to both tangent and binormal vectors with its direction determined by the right-handed system. The line $\psi(\theta)+t N(\theta)$ is the normal line at $\psi(\theta)$. Therefore, tangent vector $T(\theta)$, normal vector $N(\theta)$ and binormal vector $B(\theta)$ form a coordinate system with origin $\psi(\theta)$. The tangent line, normal line and binormal line are the three coordinate axes with positive directions given by the $T N B$ vectors, respectively. These three vectors are usually referred to as the moving trihedron or triad at point $\psi(\theta)$.

The single helical curve for a strand can be written as in vector notation by $\psi\left(\theta_{s}\right)$, $\psi\left(\theta_{s}\right)=\left(r_{s} \cos \theta_{s}, r_{s} \sin \theta_{s}, h \theta_{s}\right)$.

Vector notation of the nested helical wire centerline can be written as in vector notation by $\psi\left(\theta_{d}\right)$

$$
\begin{aligned}
\psi\left(\theta_{d}\right)= & \left(x_{s} \theta_{s}+r_{d} \cos \theta_{d} \cos \theta_{s}-r_{d} \sin \theta_{d} \sin \theta_{s} \sin \alpha_{s},\right. \\
& y_{s} \theta_{s}+r_{d} \cos \theta_{d} \sin \theta_{s}+r_{d} \sin \theta_{d} \cos \theta_{s} \sin \alpha_{s}, \\
& \left.z_{s}-r_{d} \sin \theta_{d} \cos \alpha_{s}\right),
\end{aligned}
$$

where $\theta_{d}=m \cdot \theta_{s}+\theta_{d 0}$. The tangent, binormal and normal vectors for a single helical wire and NHS can be found by substituting Eqs. (7) and (8) into Frenet-Serret formulas given in Eqs. (4) to (6) respectively. The tangent line $(\psi(\theta)+t T(\theta))$, the normal line $(\psi(\theta)+t N(\theta))$ and the binormal line $(\psi(\theta)+t B(\theta))$ can be obtained with respect to $\theta_{s}$ for single and nested helical wires, respectively. Using the definition of tangent, binormal and normal lines, three points to construct a circle on a plane can be obtained, which is perpendicular to the single helix or NH centerline.

\subsection{A Single Helical or NH Solid Part Construction}

The idea of using single helical or $\mathrm{NH}$ wire centerline to construct a solid part is based on the difficulties experienced at the modeling and analysis stages. Single helical geometry design can be easily done using the well-known 
CAD packages while the NHS is not available as a simple tool to use. NHS can be seen mostly in rope constructions at first glance. To have a NHS, a strand is wrapped over another one in a helical manner as shown in Fig. 2a. To perform the numerical analysis over this kind of structure, a fully defined model of the problem should be prepared and converted into an acceptable form for numerical analysis. Problems have been encountered at the modeling and analysis stages in different areas;

- Creating NHS is not possible by using CAD tools directly for the moment,

- Exporting NH geometry using CAD software in acceptable format for FE analysis software destroys some portion of the solid structures,

- Meshing is not possible due to irregularities on the surfaces of $\mathrm{NH}$ geometries,

- It is impossible to export $\mathrm{NH}$ geometries properly to the FE code file format and meshing the solid geometries using the FE codes encounters difficulties.

To illustrate the aforementioned problems, a single helical and $\mathrm{NH}$ geometries are modeled with the lengths of $300 \mathrm{~mm}$ and $1000 \mathrm{~mm}$ by using SolidWorks ${ }^{\circledR}$ and transferred to finite element code Abaqus/CAE ${ }^{\circledR}$ using IGES and Parasolid file formats. It should be emphasized that the procedures mentioned in this paper are evaluated by using different modeling and analysis tools such as CATIA $®$ and ANSYS ${ }^{\circledR}$ but similar results are found for the helical geometries.

Single helical parts can be generated by using the helix tool available in the library of SolidWorks and the produced single helical part can be meshed in Abaqus/CAE. Meanwhile, there is no available tool for modeling NHS in CAD packages. For this reason, NHS are modeled by using parametric equations via the explicitly written script codes for CAD packages, and then exported to FE analysis file format.

Figs. 3 and 4 have the same number of rows and columns. Column (a) represents helices created in SolidWork, columns (b) and (c) represent imported forms of IGES and Parasolid file formats in Abaqus/CAE respectively and, column (d) represents the helical geometry generated by using the parametric equations in HyperMesh $\AA$.
A new code is developed in Matlab to generate single helical/NH wire locations with the control nodes. These control nodes are exported to HyperMesh and using these control nodes, a spline is generated. Then, a normal plane perpendicular to this spline curve is generated by using the Frenet-Serret frame defined in Eqs. (4) to (6). A circle is created over this plane and swept along the single helical/NH curve centerline, which creates the meshed part in HyperMesh as shown in column (d) of Figs. 3 and 4. It has been concluded that the solid wire geometry is spoiled out when the number of control nodes and the length of wire are increased. This situation is demonstrated by comparing the cross sections of the columns (b) and (c) with item numbers (1) and (3) of Figs. 3 and 4 respectively. Comparing these figures, it can be seen that the solid structures are degenerated while the part lengths are increased from 300 $\mathrm{mm}$ to $1000 \mathrm{~mm}$. As a result, comparisons of the solid and meshed parts quality scales are given at the last columns of Figs. 3 and 4. It can be seen that the NH mesh quality is better than that of the others. This is evaluated while using $\mathrm{NH}$ mesh generated by HyperMesh in FE analysis code.

While meshing the solid parts, errors occurred in FE software due to the complex geometry of the mesh region for sweep meshing. It has been reported that the meshed parts are in unusable quality in FE analysis, which is the main problem of meshing NHS. These problems do not occur while using the proposed modeling procedure using HyperMesh. To see the problematic meshed surfaces closely, the front view of item (4) - column (b) of Fig. 4 is presented in an enlarged form in Fig. 5.

The meshed surface is degenerated and it is unusable for FE analysis. Using the proposed modeling procedure, single helical and $\mathrm{NH}$ meshed structures are created with success by using HyperMesh as in Fig. 6. It can be clearly seen that the meshed parts are precisely defined. It has been concluded that the NH meshed parts generated using this method are error free and that this procedure is the effective choice for generating helical parts for the FE analysis purposes. 


\begin{tabular}{|c|c|c|c|c|c|c|c|c|c|}
\hline \multirow{2}{*}{$\begin{array}{l}\text { Helix } \\
\text { type and } \\
\text { length }\end{array}$} & \multirow{2}{*}{$\begin{array}{l}\text { Item } \\
\text { No }\end{array}$} & \multirow{2}{*}{$\begin{array}{l}\text { Solid/Meshed } \\
\text { model }\end{array}$} & \multirow{2}{*}{$\begin{array}{l}\text { SolidWorks } \\
\text { projection } \\
\text { (a) }\end{array}$} & \multicolumn{2}{|c|}{ Abaqus/CAE imported and meshed forms } & \multirow{2}{*}{$\begin{array}{l}\text { HyperMesh model } \\
\text { exported and } \\
\text { imported in } \\
\text { Abaqus/CAE } \\
\text { (d) }\end{array}$} & \multicolumn{3}{|c|}{$\begin{array}{l}\text { Solid and Mesh Projection } \\
\text { quality }\end{array}$} \\
\hline & & & & $\begin{array}{l}\text { IGES format solid } \\
\text { and mesh } \\
\text { (b) }\end{array}$ & $\begin{array}{l}\text { Parasolid format } \\
\text { solid and mesh } \\
\text { (c) }\end{array}$ & & Low & Normal & High \\
\hline \multirow{2}{*}{$\begin{array}{c}\text { Single } \\
300 \mathrm{~mm}\end{array}$} & (1) & Solid & & & & - & & $(a, b, c)$ & \\
\hline & (2) & Mesh & - & & & & & (c) & $(b, d)$ \\
\hline \multirow{2}{*}{$\begin{array}{c}\text { Single } \\
1000 \mathrm{~mm}\end{array}$} & (3) & Solid & & & & - & $(b, c)$ & (a) & \\
\hline & (4) & Mesh & - & & & & & (c) & $(b, d)$ \\
\hline
\end{tabular}

Fig. 3. Single helix solid model created with SolidWorks and meshed using Abaqus/CAE, and model\&mesh created with HyperMesh

\begin{tabular}{|c|c|c|c|c|c|c|c|c|c|}
\hline \multirow{2}{*}{$\begin{array}{l}\text { Helix } \\
\text { type and } \\
\text { length }\end{array}$} & \multirow{2}{*}{$\begin{array}{l}\text { Item } \\
\text { No }\end{array}$} & \multirow{2}{*}{$\begin{array}{l}\text { Solid/Meshed } \\
\text { model }\end{array}$} & \multirow{2}{*}{$\begin{array}{l}\text { SolidWorks } \\
\text { projection } \\
\text { (a) }\end{array}$} & \multicolumn{2}{|c|}{ Abaqus/CAE imported and meshed forms } & \multirow{2}{*}{$\begin{array}{l}\text { HyperMesh model } \\
\text { exported and } \\
\text { imported in } \\
\text { Abaqus/CAE } \\
\text { (d) }\end{array}$} & \multicolumn{3}{|c|}{$\begin{array}{l}\text { Solid and Mesh Projection } \\
\text { quality }\end{array}$} \\
\hline & & & & $\begin{array}{l}\text { IGES format solid } \\
\text { and mesh } \\
\text { (b) }\end{array}$ & $\begin{array}{l}\text { Parasolid format } \\
\text { solid and mesh } \\
\text { (c) }\end{array}$ & & Low & Normal & High \\
\hline \multirow{2}{*}{$\begin{array}{c}\text { Nested } \\
\text { Helix } \\
300 \mathrm{~mm}\end{array}$} & (1) & Solid & & & & - & & $(a, b, c)$ & \\
\hline & (2) & Mesh & - & & & & $(b, c)$ & & (d) \\
\hline \multirow{2}{*}{$\begin{array}{l}\text { Nested } \\
\text { Helix } \\
1000 \mathrm{~mm}\end{array}$} & (3) & Solid & & & & - & $(b, c)$ & (a) & \\
\hline & (4) & Mesh & - & & & & $(b, c)$ & & (d) \\
\hline
\end{tabular}

Fig. 4. NHS solid models created with SolidWorks and meshed with Abaqus/CAE, and NHS modeled and meshed with HyperMesh 


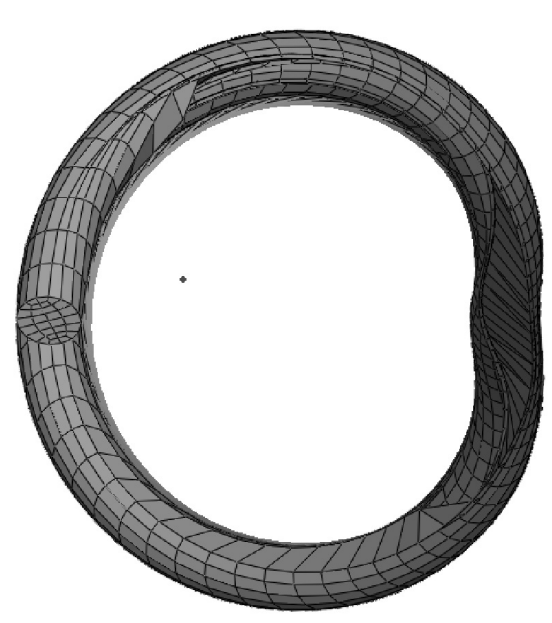

Fig. 5. Zoomed front view of a $\mathrm{NH}$ wire

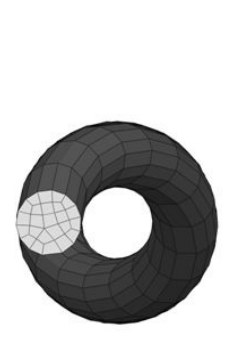

a)

Fig. 6. a) single helical, b) NH meshed solid parts in Abaqus/CAE

\subsection{Construction of a Complex Single Helical and NH Wire Mesh}

During the modeling issue, it was observed that finite element software needs smooth and precisely defined solid meshed surfaces. Meanwhile, the commercial CAD package is not suitable for the construction of $\mathrm{NH}$ geometry because of the irregularities that appeared at the meshing stage. FE analysis code needs precisely meshed solid models. Therefore, the idea of designing a meshed model of the single helical and $\mathrm{NH}$ wire is proposed as a solution. An algorithm to develop a meshed model of a single or NH wire is presented in Fig. 7. The algorithm includes mainly four stages:

- geometry generation,

- solid Part and Mesh generation,
- model generation,

- analysis of the result processing.

In the geometry generation stage, a new code is developed to find the single helical or $\mathrm{NH}$ control nodes using Eq. (1) or Eqs. (2) and (3), respectively. To construct a solid geometry, a normal plane which is perpendicular to the single helical/NH spline curve is needed. Frenet-Serret frame is constructed over the single helix/NH curve using the Eqs. (4) to (6) by substituting Eqs. (7) and (8) respectively. Using the Frenet-Serret equations tangent, normal and binormal lines as depicted in Fig. 2a are defined and three points are generated to construct a plane perpendicular to the helical spline curve using these points. At the end of the geometry generation stage, the control nodes to construct single/ $\mathrm{NH}$ wire and the nodes to build a plane perpendicular to the single helical/ $\mathrm{NH}$ wire is output to a file in "*.inp" file extension as shown in Fig. 8.

Solid part and mesh generation stages compose the main parts of wire strand/rope generation procedure. The "*.inp" file, which holds the helical geometry, is imported into the HyperMesh. A spline is constructed using the control nodes corresponding to the single/ NH wire. The normal plane defined by FrenetSerret formulae is used to create a circle, which is perpendicular to the helical wire centerline. The surface of this 2D circle geometry is divided by quadratic brick elements of type C3D8R, which are dragged along the helical wire path to construct a meshed single helical/NH wire model. The generated shape is constituted with meshes and evaluated as orphan mesh in Abaqus/CAE. At the end of this operation, meshed helical wire geometry is exported to finite element model structure with "*.inp" file format, which can be imported by Abaqus/CAE.

In the model generation stage the orphan mesh geometry is imported in Abaqus/CAE which is constructed by using HyperMesh as mentioned above. It can be concluded that the wires developed by using the proposed method are well defined without length limitation and free of surface irregularities. While defining the contacts between wires using the generated meshes in IGES/Parasolid file formats, errors are encountered because of the conflicts between the wires due to irregular meshed surfaces. The new 


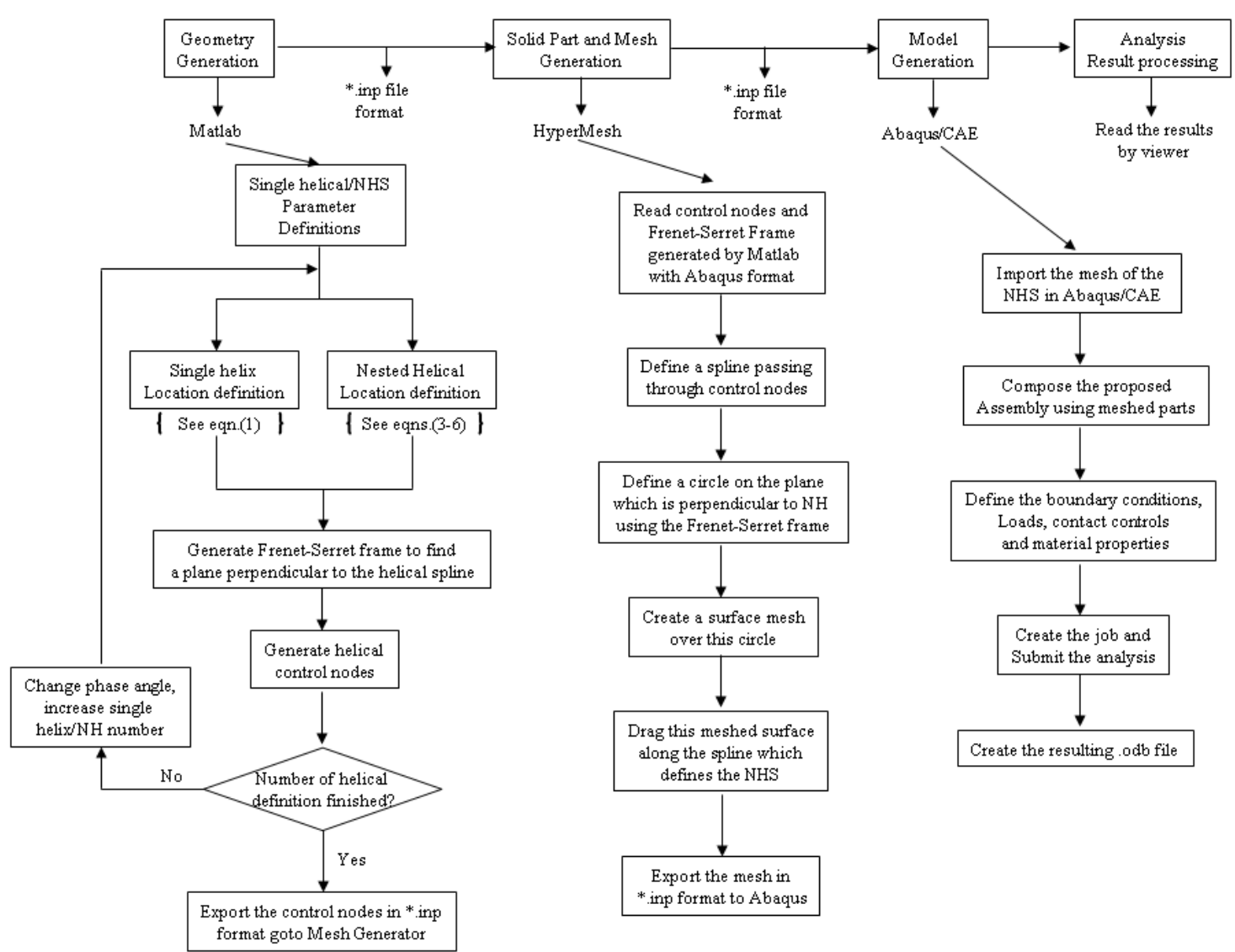

Fig. 7. Generating the NHS model and analysis algorithm

NH meshed model gets rids of such problems, defining precise surfaces. Analysis stage can be conducted with the produced mesh of the wire strand/rope model.

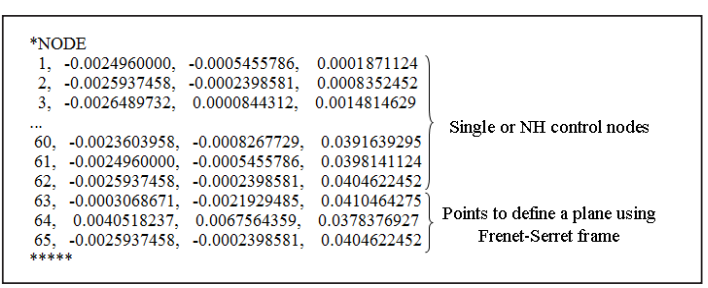

Fig. 8. Geometry definition in Abaqus/CAE file

\section{format}

After the solid part and mesh generation stage, assembly of the simple straight strand is composed by wrapping six single helical wires around a straight wire. Then, a single outer helical wire and six $\mathrm{NH}$ wires surrounding it are imported.
This composes an outer strand of an IWRC, which is assembled around the center straight strand to compose the complete final wire rope model shown in Fig. 9. It can be clearly seen that the wire rope structure includes $36 \mathrm{NH}$ wires, which makes the FE analysis complicated. The number of nodes and elements also exceeds 200,000. Boundary conditions, load definitions, material properties, and contact controls are defined in Abaqus/CAE and the created job is submitted for the analysis of the proposed problem. Finally, the FE analysis result file is processed to produce numerical results for the solved problem using Abaqus/CAE viewer.

\section{FINITE ELEMENT ANALYSIS OF THE WIRE STRAND/ROPE MODEL}

In this section, an axial wire strand/rope FE analysis stage is presented. Material properties are defined based on the previous study of Jiang [9]. 


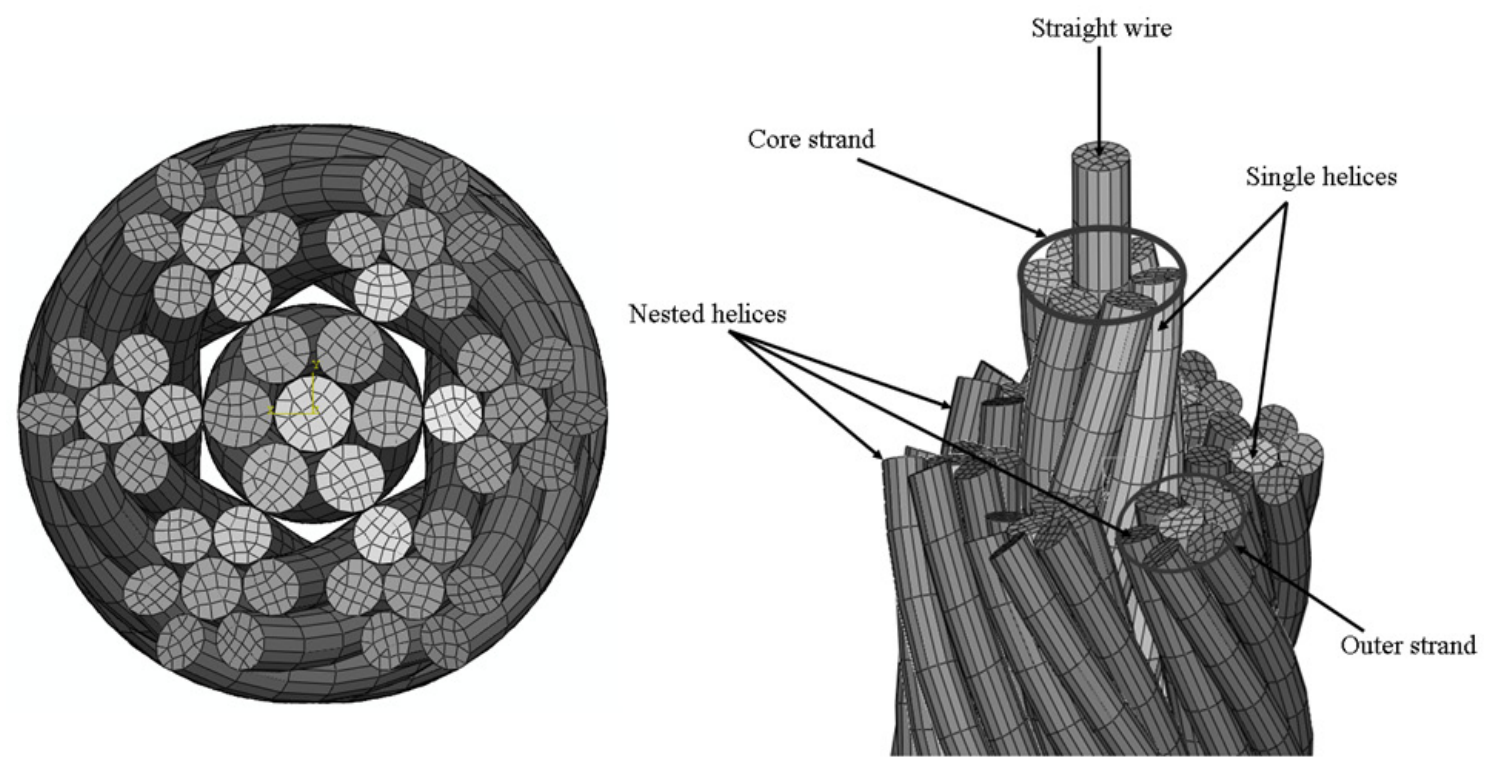

Fig. 9. A left Lang lay meshed wire rope structure

During the FE analysis models 8 nodes quadratic brick elements of type C3D8R are used. One side of the model is constraint to be a fixed boundary condition, while the other side is constraint not to rotate only in $z$ axis. A friction coefficient of $\mu=0.115$ is defined. An explicit analysis is conducted over the model.

A $28.75 \mathrm{~mm}$ length $(6+1)$ Wire Strand (WS) is analyzed first with $R_{1}=1.97 \mathrm{~mm}, R_{2}=1.865 \mathrm{~mm}$ and pitch length is $p_{2}=115 \mathrm{~mm}$. Surface to surface contact is defined between center and six helical wires and between individual helical wires by considering the frictional effects. An axial strain of $\varepsilon=0.03$ is applied to the non-rotating end of the model. Finite element analysis results show that a helical line of contact occurs between the center wire and the outer helical wires, and between consecutive helical wires of the strand as shown in Fig. 10a. The numbers of 42,818 C3D8R type elements are used in Fig. 10a. The mesh is refined to 196,524 number of C3D8R type elements and a more smooth helical line of contact between wires are obtained as shown in Fig. 10b.

As a second example, $300 \mathrm{~mm}$ length 6 $\times 7$ Right Lang Lay (RLL) Independent Wire Rope Core IWRC is modeled, using the proposed modeling procedure. Wire radiuses are defined as $R_{1}=1.97 \mathrm{~mm}, R_{2}=1.865 \mathrm{~mm}, R_{3}=1.6 \mathrm{~mm}$, $R_{4}=1.5 \mathrm{~mm}$, pitch lengths are defined as $p_{2}=70$ $\mathrm{mm}, p_{4}=193 \mathrm{~mm}$ and $p_{2}{ }^{*}=70 \mathrm{~mm}$. An axial strain of $\varepsilon=0.015$ is applied to the non-rotating end of the model. Wire by wire based FE analysis results are presented in Fig. 11. Analysis shows that the core wire of the IWRC holds the most of the axial force among the wires within an IWRC. In addition, the core strand of the IWRC encounters the maximum amount of load distribution during the axial loading process.

These illustrative examples show that the proposed modeling and analysis procedure give the desired information about the behavior of wires within a wire rope. The proposed modeling scheme can be developed to solve more difficult wire rope problems, such as wire rope bent over sheave. From this point of view, it would be worth improving the present study for further use.

\section{CONCLUSIONS}

A new methodology and algorithm of constructing single and nested helical geometries are described in this paper. The problems faced during the model generation and meshing stages of helical structures are described and the solution strategies are argued. Instead of modeling a single helical or NH solid structure and meshing, it is proposed to model them directly as an orphan mesh by using the parametric mathematical 


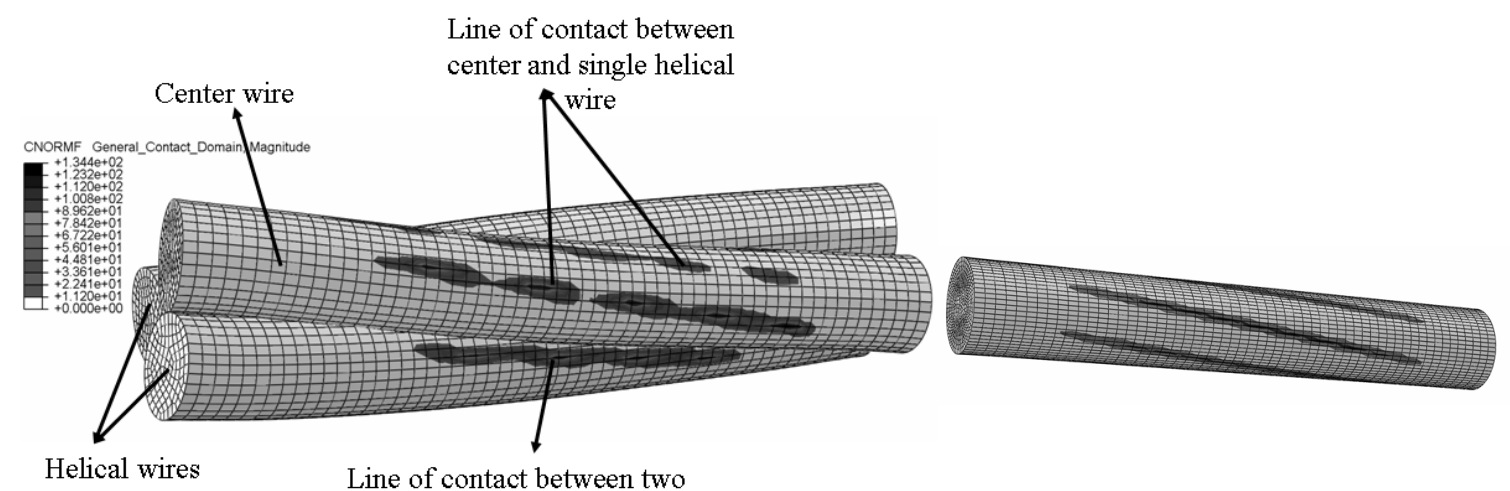
consecutive single helical wires

a)

b)

Fig. 10. a) contact force distribution over a simple straight strand, b) line of contact over the center wire with a fine mesh

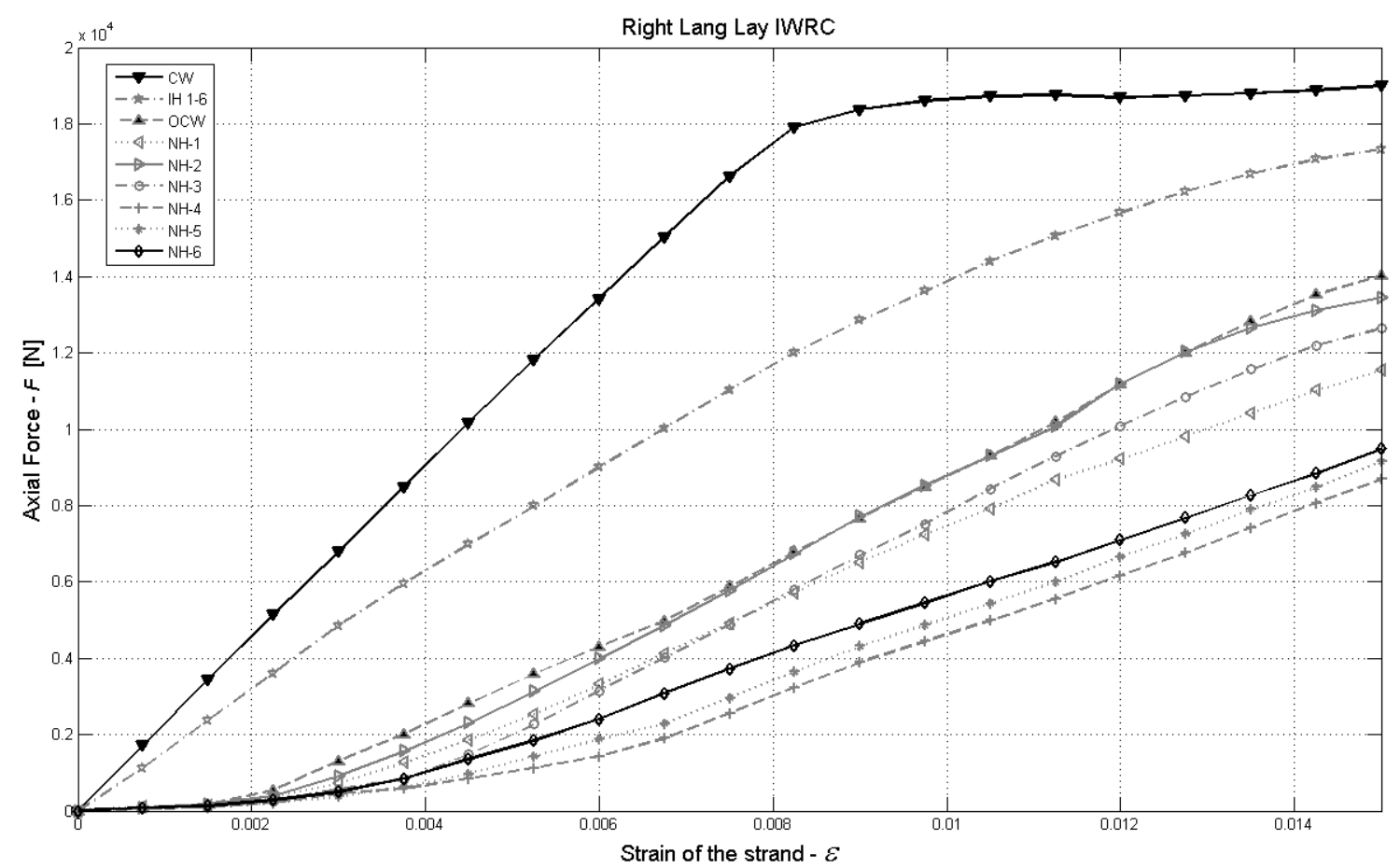

Fig. 11. A right Lang lay $6 \times 7$ IWRC $300 \mathrm{~mm}$, axial force-strain FE analysis result

definitions of the single helical and NHS. In this manner, problems encountered in the surfaces of the helical wires are discarded at the source of the modeling stage. The proposed procedure for modeling is presented and discussed with illustrative examples. This process removes the surface irregularities encountered over the complex helical structures and generates precise geometries. Data losses are obstructed while transferring solid parts between CAD package and finite element software by this proposed modeling strategy and algorithm. At the same time, the length limitations are left behind. The proposed procedure creates precise geometries without length limitations at all. Finally, FE analysis examples are presented to show the benefits of 
the proposed modeling scheme. A wire strand is analyzed using the axial loading problem. The results of FE analysis show that there is a helical line of contact between wires within a strand. Another example is solved on a $6 \times 7$ wire RLL IWRC. Wire by wire axial force distribution is graphed by using the results of the FE analysis, which gives detailed information about the behavior of the wires within an IWRC. These examples demonstrate the benefits of FE analysis using the proposed modeling scheme. Further studies can be conducted by using the proposed modeling procedure to obtain detailed information about helical structures. In addition, the present modeling technique can be developed and used for further studies.

\section{REFERENCES}

[1] Metzler, R., Dommersnes, P.G. (2004). Helical packaging of semiflexible polymers in bacteriophages. European Biophysics Journal, vol. 33, no. 6, p. 497-505.

[2] Meng, X., Li, J., Hou, H., Song, Y., Fan, Y., Zhu, Y. (2008). Double helix chain frameworks constructed from bisbenzotriazole building blocks: Syntheses, crystal structures and third-order nonlinear optical properties. Journal of Molecular Structure, vol. 891, no. 1-3 , p. 305-311.
[3] Luo, F., Che, Y., Zheng, J. (2006). Synthesis and description of the first helical chain of $\mathrm{Cu}-\mathrm{Pb}$ bimetallic atoms and $\mathrm{Cu}(\mathrm{I})-\mathrm{Cu}(\mathrm{II})$ mixed-valence. Inorganic Chemistry Communications, vol. 9, p. 848-851.

[4] Costello, G.A., Sinha, S.K. (1977). Static Behaviour of Wire Rope. Proceedings ASCE, Journal of Engineering Mechanical Division, vol. 103, no. EM6, p. 1011-1022.

[5] Velinsky, S.A. (1993). A stress based methodology for the design of wire rope systems. Transactions of ASME, Journal of Mechanical Design, vol. 115, p. 69-73.

[6] Velinsky, S.A. (1988). Design and mechanics of multi-lay wire strands. Transactions of ASME, Journal of Mechanics, Transmissions, and Automation in Design, vol. 110, p. 152-160.

[7] Frey, J.P., George, P.-L. (2000). Mesh Generation application to finite elements. Hermes Science Publishing, Oxford.

[8] Elata, D., Eshkenazy, R., Weiss, M.P. (2004). The mechanical behavior of a wire rope with an independent wire rope core. International Journal of Solids and Structures, vol. 41, p. 1157-1172.

[9] Jiang, W.G., Henshall, J.L. (1999). The analysis of termination effects in wire strand using finite element method. Journal of Strain Analysis, vol. 34, no. 1, p. 31-38. 